\title{
Comparison of Fluoride Ion Concentration in Black, Green and White Tea
}

\author{
Research Article
}

Mahsa Esfehani $^{1^{*}}$, Shabnam Ghasemzadeh ${ }^{2}$, Monirsadat Mirzadeh $^{3}$

\section{Assistant Professor, Dental Caries Prevention Research Center, Faculty of Dentistry, Qazvin University of Medical Sciences, Qazvin, Iran.}

2. General Dentist, Dental Caries Prevention Research Center, Qazvin University of Medical Sciences, Qazvin, Iran. 3. Assistant Professor of Metabolic Disease Research Center, Qazvin University of Medical Sciences, Qazvin, Iran.

\begin{abstract}
Back ground: Tea is one of the most commonly used drinks in the world. Drinking too much tea might affect the health. Tea is known for its fluoride resources; hence, consuming tea especially together with other fluoride resources can increase the risk of appearance of dental fluorosis. Considering the unspecified concentration of fluoride in white tea in Iran, the current study has carried out to evaluate the concentration of fluoride in different types of black tea, green tea and white tea of Refah Factory of Lahijan. Method and Material: In this study, the content of the fluoride of the black, green and white teas were measured. One gram samples of each type of tea were provided. Each time, the samples were placed in 200 milliliters of boiling distilled water, then it was moved to 80 degrees of Celsius incubator to be brewed. After 5 and 15 minutes, the solution was filtered. Concentration of the fluoride was measured through chromatography method and was recorded by measure of milligrams per liter. Data were analyzed using SPSS21 software. And significant level of less than 0.05 was considered. Findings: The concentration of the fluoride of the black, green and white solutions at the time of 5 minutes of brewing were respectively $1.38,1.07$ and 0.18 milligrams per liter and significantly increased he concentration of fluoride in all three types of tea with increasing drainage time ( $p$ value $<0.05)$. Conclusion: The concentration of the fluoride of the three tea types were significantly different, to the extent that the least fluoride concentration belonged to the white and the most concentration belonged to black tea at both brewing times. By increasing the time of brewing the tea, its concentration was increased.
\end{abstract}

\section{Keywords: Fluoride, Black Tea, Green Tea, White Tea, Chromatography}

\section{Introduction}

Tea is a drink which is made from the leaves of the Camellia sinensis plant and one of the most popular beverages among the people. About three million kilograms of tea is produced annually around the word and consumed. Of the tea produced in the world, $78 \%$ is black tea which is commonly used in western countries, $20 \%$ of the tea is green that is commonly used in Asian countries which is manly provided in China and Japan and although recently, there are many recommendations for taking this type of tea(1) (2). The tea plant can optionally absorb the fluoride of the soil and store it in its organs (3). That $98 \%$ of fluoride stored in tea is stored in its leaves. The amount of fluoride increases with leaf maturity. There are $4-100 \mathrm{mg} / \mathrm{kg}$ of fluoride in young buds and young leaves that usually provides green or black tea. In addition factors such as soil acidity, Oxidation rate, processing method, plant age and etc. that are effective in the amount of fluoride in tea leaves, other factors such as water temperature, water hardness, and various times of tea brewing are effective in reducing fluoride intake during preparation (4). As well as various types of tea, different amounts of

*Corresponding Author:

Mahsa Esfehani

Assistant Professor,

Department of Oral and Maxillofacial Medicine,

Faculty of Dentistry,

Qazvin University of Medical Sciences, Qazvin, Iran

Email id: esfahani ma@yahoo.com fluoride are different (5). Fluoride is now the cornerstone of modern preventive dentistry, in addition, it is used to treat Osteoporosis, ischemia and other major diseases (6). The amount of fluoride entering the body should be controlled in order to prevent fluoride from being overused, including fluorosis and fluoride poisoning. Fluoride supply sources include drinking water, fluoride- containing salts in foods, fluoridecontaining beverages, including tea, toothpastes, and other oral health products (7). In the study of Quack, tea in children is considered as a primary source of hydration and increases the risk of inflammation of the child to fluorosis (8). Also, in the study of Koblar, 5 cups of tea can suffice for 9 to $10 \%$ of the fluoride requirement of a $70 \mathrm{~kg}$ adult (9). Since tea is considered as a source of fluoride, so people who drink a lot of tea or use a variety of high fluoride tea, are at risk of developing dental fluorosis (10). This issue becomes even more important when absorbing fluoride from tea consumption with other fluoride sources, including fluoride from drinking water (11). Therefore, controlling the quality of tea as an important source of fluoride is important. Since drinking tea is very common among Iranians, due to the uncertainty about the current fluoride content of tea in our country, the present study aimed to determine the concentration of fluoride in black, green and white tea in Iran. Since fluoride is substantially consumed through tea drinking, it is imperative that tea factories around the world provide consumers with information about the concentration of tea fluoride. Considering the world Health Organization`s recommendation that maximum fluoride intake should 
be used (0.07- $0.05 \mathrm{mg} / \mathrm{l}$ per kilogram of body weight per day, up to a maximum of $4 \mathrm{mg}$ in Adults and $2 \mathrm{mg}$ in children. And given that in Iran, about 31 to 38 percent of fluoride is consumed through tea consumption $(15,21)$

\section{Materials}

This research is a cross-sectional (laboratory) study that examines all kinds of black tea, white tea and green tea factory in Lahijan. The types of tea mentioned were purchased with less than one month's production date difference. We first weighed 100 grams of black, green and white tea. Each $100 \mathrm{~g}$ was divided into 4 equal parts, then $10 \mathrm{~g}$ of each section were removed randomly. Samples are spread in squares $10 * 10 \mathrm{~cm}$. approximately 20 gr from 4 regions were selected and finally prepared with a precision of $0.1 \mathrm{mg}, 12$ samples of 1 frame of each type of tea. The specimens were then placed in an oven for 4 hours at $60^{\circ} \mathrm{C}$ to obtain their moisture, and then the specimens were placed in a desiccator to dry, for each sample, $200 \mathrm{ml}$ of Merck, Germany, ultra-pure water was placed to dry, for each sample, $200 \mathrm{ml}$ of deionized water (Merk , Germany) ultra - pure was placed on a flame in a jellyfish balloon. After the water was boiled, a $1 \mathrm{~g}$ sample was added and immediately transferred to an incubator at $80^{\circ} \mathrm{C}$. On each balloon, a plate was placed in order to prevent the evaporation of water. After 5 minutes, samples of black tea, green and white were removed. Other samples were prepared individually to prevent opening the incubator door and placed inside the incubator at $80^{\circ} \mathrm{C}$ and after 15 minutes, it was removed from the incubator. This was done for each tea for 6 minutes for 5 minutes and 6 times for 15 minutes. Until the beginning of the analysis, the specimens were kept inside the eagle in a closed container at $4{ }^{\circ} \mathrm{C}$. It should be noted that the tea sample can remain at this temperature for up to 48 hours before the analysis. Finally, the concentration of fluoride ion in $\mathrm{mg} / \mathrm{l}$ or $\mathrm{ppm}$ was calculated and the data were analyzed using SPSS21 statistical software and t-test, 'ANOVA and post hoc tests.

\section{Findings}

The highest mean fluoride concentration was observed in both black and white tea for 5 and 15 minutes (1.38 and $2.31 \mathrm{mg} / \mathrm{l}$ respectively). According to the results shown in Table 1 , the concentration of fluoride in all three types of tea increased with increasing drainage time and this difference was significant ( $\mathrm{p}$ value $<0.05$ )

Table 1: Comparison of fluoride concentration in all three types of tea (in $\mathrm{mg} / \mathrm{l}$ ) at the time of brewing 5 and 15 minutes.

\begin{tabular}{|c|c|c|c|c|}
\hline Kind of tea & brewing time & Mean & Standard deviation & p-value \\
\hline \multirow{2}{*}{ White tea } & 5 & 1.07 & 0.03 & \\
& 15 & 1.57 & 0.05 & \multirow{2}{*}{0.001} \\
\hline \multirow{2}{*}{ Black tea } & 5 & 1.38 & 0.03 & \\
\cline { 2 - 4 } & 15 & 2.31 & 0.05 & \\
\hline \multirow{2}{*}{ Green tea } & 5 & 0.18 & 0.00 & \\
\cline { 2 - 5 } & 15 & 0.29 & 0.01 & \\
\hline
\end{tabular}

Mean concentration of fluoride ion in three types of tea was significantly different in 5 minutes (p value $<0.001)$ after post following tests, the mean fluoride ion concentration also showed a significant different between all three types of tea (pvalue $<0.001$ ).

\section{Discussion}

In the present study, the concentration of fluoride in different typed of black, green and white tea in the course of 5 minutes, the breeches are arranged was 1.07 , $1.38,0.18 \mathrm{mg} / \mathrm{l}$ and in the course of 15 minutes, the breeches are arranged was $1.57,2.31,0.29 \mathrm{mg} / \mathrm{l}$. in both breaks, the lowest was white tea and most of it was black tea.

In the study Malinowska the concentration of white tea fluoride ion was $0.37,0.54$ and green tea was $62.21-1.0 \mathrm{mg} / \mathrm{l}$ and black tea was $4.54-0.32 \mathrm{mg} / \mathrm{l}$. The results of these studies, which were conducted on a variety of tea and vegetable beverage in Poland, except for the concentration of which tea fluoride which is slightly different from the slightly different from the results of the present study, in other cases, it matched. This difference can be attributed to the different in the time taken for harvesting white tea, the cultivation region and its different quality (11).

In the Rote study, the concentration of green tea fluoride ion, which had been brewing for 5 minutes, was reported to be $0.8,2,0 \mathrm{mg} / 1$ (12).

In the Cao study, the concentration of black tea fluoride $0.94-1.41 \mathrm{mg} / \mathrm{l}$ was consistent with the current study (13). Based on the results of this study, the difference in the concentration of fluoride ion in black, green and white packaging was statistically significant. Considering that one gram of tea leaf was weighed out to be more accurate for each item, and given that the sheets of all three tea are made from Camilla cyssine, the difference is due to the quality of the tea leaf, which leaves the white tea is the most delicious and sweetest leaf of the plant.

Also difference can also be attributed to the length of time the tea leaves reach the mature plant, with increasing maturity of leafy plants, fluoride levels increase and its quality is reduced. In addition to the above, factors such as soil acidity, oxidation and processing in fluoride in tea leaves are effective (16). There are other factors that influence the amount of fluoride release during tea preparation: water temperature (studies show that increasing water temperature while brewing tea will significantly increase the release of fluoride) and the degree of water hardness (increasing water hardness reduces fluoride extraction during the preparation process) (16)(17). 
Other causes of difference in concentration studies include different genetic variations of the plant, variation in tea harvest time, soil compositions, rainfall, specificity and plant height (7). In the study, which measured the concentration of tea fluoride after 5, 10 and 30 minutes of drainage, with increasing drainage time, tea drink fluoride concentration increased significantly (13).

Zerubruk measured the concentration of fluoride released in the black tea and green tea drink at times of $3,5,10$, the results indicated an increase in fluoride content due to increased drainage time, the results indicated an increase in fluoride content due to increased drainage time (17). Also, in the study, the fluoride concentration increased with the increase in the duration of tea withdrawal. In this study, with increasing drainage time, fluoride concentration increased (18). It should be noted that the concentration of tea fluoride ion can be measured by selective ion electrode and chromatography. In studies, Maleki \& Reto chromatography method and in studies, Amanluo, Zerubruk and Esfahanizadeh, an ion selective electrode method was used to measure the fluoride concentration of tea types $(14,21,17,20,19)$. In the present study, chromatography, the latest and most reliable method for measuring fluoride ion, was used.

Different studies pointing to the link between increasing the risk of fluorosis and recommending an increase in tea intake. In area where fluoride is poor, it is recommended to use black tea with the highest concentration of fluoride, and also to use a variety of tea has been cooked for more time. Considering the maximum daily fluoride intake $(2 \mathrm{mg}$ children, $4 \mathrm{mg}$ adults) approximate daily consumption of 8 grams of white tea, 1.5 grams of green tea and 1 gram of black tea. Lahigan welfare can provide about 50 percent of the daily allowable fluoride intake for adults and all allowable amounts of food for children.

\section{References}

1. Fulian Y. Discussion on the Originating Place and the Originating Center of Tea Plant [J]. Journal of Tea Science. 1986;1;000.

2. Wiseman SA, Balentine DA, Frei B. Antioxidants in tea. Critical Reviews in Food Science \& Nutrition. 1997 Dec 1;37(8);705-18.

3. Cao J, Bai X, Zhao Y, Liu J, Zhou D, Fang S, Jia M, $\mathrm{Wu}$ J. The relationship of fluorosis and brick tea drinking in Chinese Tibetans. Environmental health perspectives. 1996 Dec;104(12);1340.

4. Kalayc1 S, Somer G. Factors affecting the extraction of fluoride from tea: Application to three tea samples. Fluoride. 2003;36(4);267-70.

5. Fung KF, Zhang ZQ, Wong JW, Wong MH. Aluminium and fluoride concentrations of three tea varieties growing at Lantau Island, Hong Kong. Environmental Geochemistry and Health. 2003 Jun $1 ; 25(2) ; 219-32$.

6. Emekli-Alturfan E, Yarat A, Akyuz S. Fluoride levels in various black tea, herbal and fruit infusions consumed in Turkey. Food and chemical toxicology. 2009 Jul 1;47(7);1495-8.
7. Mojarad F, Khanlary E. Assessment of Fluoride Levels in Different Brands of Black and Green Tea Consumed in Iran. Scientific Journal of Hamadan University of Medical Sciences. 2013 Jan 1;19(4);3642.

8. Quock RL, Gao JX, Chan JT. Tea fluoride concentration and the pediatric patient. Food chemistry. 2012;130(3);615-7

9. Koblar A, Tavčar G, Ponikvar-Svet M. Fluoride in teas of different types and forms and the exposure of humans to fluoride with tea and diet. Food Chemistry. 2012;130(2);286-90

10.Lung SC, Cheng HW, Fu CB. Potential exposure and risk of fluoride intakes from tea drinks produced in Taiwan. J Expo Sci Environ Epidemiol 2008; 18;15866.

11. Malinowska E, Inkielewicz I, Czarnowski W, Szefer P. Assessment of fluoride concentration and daily intake by human from tea and herbal infusions. Food Chem Toxicol 2008;46; 1055-61.

12. Reto M, Figueira ME, Filipe HM, Almeida CM. Chemical composition of green tea (Camellia sinensis) infusions commercialized in Portugal. Plant foods for human nutrition. 2007;62(4);139.

13. Cao J, Zhao Y, Li Y, Deng HJ, Yi J, Liu JW. Fluoride levels in various black tea commodities: measurement and safety evaluation. Food and Chemical Toxicology. 2006; 44(7);1131-7.

14. Shu W, Zhang Z, Lan C, Wong M. Fluoride and aluminium concentrations of tea plants and tea products from Sichuan Province, PR China. Chemosphere. 2003;52(9);1475-82.

15. Hayacibara MF, Queiroz CS, Tabchoury CPM, Cury JA. Fluoride and aluminum in teas and tea-based beverages. Revista de Saude Publica. 2004;38(1);1005.

16.Zerabruk S, Chandravanshi BS, Zewge F. Fluoride in black and green tea (Camellia sinensis) infusions in Ethiopia: measurement and safety evaluation. Bulletin of the Chemical Society of Ethiopia. 2010;24(3).

17.Kyle JA, Morrice PC, McNeill G, Duthie GG. Effects of infusion time and addition of milk on content and absorption of polyphenols from black tea. Journal of Agricultural and Food Chemistry. 2007;55(12);488994.

18. Amanlou M, Nabati F, Azizian H, Farsam H. Assessment of fluoride content and daily intake from different brands of tea bags in Iran. Research in Pharmaceutical Sciences. 2009;3(2);55-9.

19. Esfahanizadeh K, Amanloo M, Valaee N, Zahed SM. The Fluoride Content of Some Frequently Used Teabags in Iran. Journal of Dentistry, Shiraz University of Medical Sciences. 2010;11(2);169-72.

20. Maleki A, Daraei H, Mohammadi E, Zandi S, Teymouri P, Mahvi AH, et al. Daily Fluoride Intake from Iranian Green Tea: Evaluation of Various Flavorings on Fluoride Release. Environmental health insights. 2016;10;59-63.

21.Lung SC, Hsiao PK, Chiang KM. Fluoride concentrations in three types of commercially packed tea drinks in Taiwan. Journal of exposure analysis and environmental epidemiology. 2003;13(1);66-73. 